\title{
A Ferroptosis-Related Gene Prognostic Index Associated With Biochemical Recurrence and Radiation Resistance for Patients With Prostate Cancer Undergoing Radical Radiotherapy
}

Dechao Feng ${ }^{\dagger}$, Xu Shit, Qiao Xiong, Facai Zhang, Dengxiong Li, Wuran Wei and Lu Yang *

Department of Urology, Institute of Urology, West China Hospital, Sichuan University, Chengdu, China

Background: Ferroptosis is a new type of programmed cell death which has been reported to be involved in the development of various cancers. In this study, we attempted to explore the possible links between ferroptosis and prostate cancer (PCa), and a novel ferroptosis-related gene prognostic index (FGPI) was constructed to predict biochemical recurrence $(\mathrm{BCR})$ and radiation resistance for $\mathrm{PCa}$ patients undergoing radical radiotherapy (RRT). Moreover, the tumor immune microenvironment (TME) of PCa was analyzed.

Methods: We merged four GEO datasets by removing batch effects. All analyses were conducted with $R$ version 3.6.3 and its suitable packages. Cytoscape 3.8.2 was used to establish a network of transcriptional factor and competing endogenous RNA.

${ }^{\dagger}$ These authors have contributed equally to this work and share first authorship

Specialty section:

This article was submitted to Cell Death and Survival, a section of the journal Frontiers in Cell and Developmental Biology

Received: 28 October 2021 Accepted: 04 January 2022 Published: 10 February 2022

Citation:

Feng $D$, Shi $X$, Xiong $Q$, Zhang F, Li D, Wei $W$ and Yang $L$ (2022) A Ferroptosis-Related Gene Prognostic Index Associated With Biochemical Recurrence and Radiation Resistance for Patients With Prostate Cancer Undergoing Radical Radiotherapy. Front. Cell Dev. Biol. 10:803766. doi: 10.3389/fcell.2022.803766
Results: We established the FGPI based on ACSL3 and EPAS1. We observed that FGPI was an independent risk factor of BCR for PCa patients (HR: 3.03; 95\% Cl: 1.68-5.48), consistent with the result of internal validation (HR: 3.44; 95\% Cl: 1.68-7.05). Furthermore, FGPI showed high ability to identify radiation resistance (AUC: 0.963; 95\% Cl: 0.882-1.00). LncRNA PART1 was significantly associated with BCR and might modulate the mRNA expression of EPAS1 and ACSL3 through interactions with 60 miRNAs. Gene set enrichment analysis indicated that FGPI was enriched in epithelial-mesenchymal transition, allograft rejection, TGF beta signaling pathway, and ECM receptor interaction. Immune checkpoint and m6A analyses showed that PD-L2, CD96, and METTL14 were differentially expressed between BCR and no BCR groups, among which CD96 was significantly associated with BCR-free survival (HR: 1.79; 95\% Cl: 1.06-3.03). We observed that cancer-related fibroblasts (CAFs), macrophages, stromal

\footnotetext{
Abbreviations: APCs, antigen-presenting cells; BCR, biochemical recurrence; CAF, cancer-associated fibroblast; ceRNA, competing endogenous RNA; CRPC, castration-resistant prostate cancer; GEO, Gene Expression Omnibus; GPX4, glutathione peroxidase; GSH, glutathione; lncRNA, long noncoding RNA; mRNA, messenger RNA; NK cells, natural killer cells; PCa, prostate cancer; RP, radical prostatectomy; RRT, radical radiotherapy; SRT, salvage radiotherapy; TAMs, tumor-associated macrophages; TCGA, The Cancer Genome Atlas; TME, tumor immune microenvironment.
} 
score, immune score, estimate score, and tumor purity were differentially expressed between BCR and no BCR groups and closely related to BCR-free survival (HRs were $2.17,1.79,2.20,1.93,1.92$, and 0.52 for cancer-related fibroblasts, macrophages, stromal score, immune score, estimate score, and tumor purity, respectively). Moreover, cancer-related fibroblasts (coefficient: 0.20), stromal score (coefficient: 0.14), immune score (coefficient: 0.14), estimate score (coefficient: 0.15), and tumor purity (coefficient: -0.15) were significantly related to FGPI, among which higher positive correlation between cancer-related fibroblasts and FGPI was observed.

Conclusion: We found that FGPI based on ACSL3 and EPAS1 might be used to predict $\mathrm{BCR}$ and radiation resistance for PCa patients. CD96 and PD-L2 might be a possible target for drug action. Besides, we highlighted the importance of immune evasion in the process of BCR.

Keywords: ferroptosis-related gene prognostic index, prostate cancer, tumor immune microenvironment, biochemical recurrence, immune checkpoint

\section{INTRODUCTION}

Prostate cancer (PCa) has been the most common non-skin malignant tumor among American men since 1984, accounting for $26 \%$, and the second leading cause of cancer deaths (Siegel et al., 2021). Radical treatment is one of the most common treatments for localized $\mathrm{PCa}$, which mainly includes radical prostatectomy (RP) and radical radiotherapy (RRT). However, despite the improvement of techniques and perfect selection of indications for patients, $27-53 \%$ of men still encounter biochemical recurrence (BCR) (Amling et al., 2000; Hull et al., 2002; Roehl et al., 2004; Van den Broeck et al., 2019). Moreover, although thought to be a slowly growing tumor, about $10-70 \%$ of PCa patients experience recurrence after receiving RRT, indicating that some subsets of PCa cells possess radiation resistance (Allen et al., 2007; Weber et al., 2009). For recurrence patients, the median time to metastasis is 8 years, and the median time from metastasis to death is 5 years (Pound et al., 1999). In the most recent update, the median time for metastasis-free survival was 10 years (Antonarakis et al., 2012). Once a recurrence occurs, management of the tumor often becomes tricky. Thus, early prediction helps the realization of individualized precision medicine. At present, Gleason score is the most important prognostic factor that affects the biological behavior of tumors and predicts patient response to treatment (Epstein et al., 2016). D'Amico et al. proposed in 2000 that the percentage of positive prostate biopsies added clinically significant information regarding time to failure of prostatespecific antigen (PSA) after RP along with Gleason score, preoperative PSA value, and staging together. However, there is still no good standard to individually predict radiotherapy resistance population and high-risk BCR population.

Ferroptosis is a new type of programmed cell death defined in 2012 as different from necrosis and apoptosis, which may be caused by metabolic stress, such as glutathione (GSH) depletion, and is characterized by the accumulation of iron-dependent lipid peroxides to a lethal level (Stockwell et al., 2017). The ferroptosisrelated enzyme GSH peroxidase 4 (glutathione peroxidase,
GPX4) is the only enzyme that can use GSH as an electron donor to reduce the toxic lipid hydroperoxide in the biofilm to the corresponding alcohol (Yang et al., 2014; Rohr-Udilova et al., 2018). While GSH reduces reactive oxygen species and reactive nitrogen species, which can induce the polyunsaturated fatty acids in the phospholipids of the biomembrane to produce a lipid peroxidation chain reaction, it causes significant peroxidative damage and cell death (Das, 2019). Therefore, both the depletion of GSH and the decrease of GPX4 activity will cause the accumulation of lipid peroxides in the cells, thereby promoting the ferroptosis process. Overexpression of GPX4 in PCa cells inhibits cell cycle progression and cell migration by increasing GSH and reducing levels of reactive oxygen species, thereby inhibiting PCa progression (Rohr-Udilova et al., 2018). Simultaneously, GPX4 is the main target of ferroptosis inducers. Erastin can inhibit the activity of GPX4 by consuming glutathione, while RSL3 can directly inhibit the activity of GPX4 (Shimada et al., 2016; Jelinek et al., 2018). It was recently discovered that a second glutathione-independent protective pathway, FSP1/AIFM2, works in parallel with GPX4 during ferroptosis (Bersuker et al., 2019; Doll et al., 2019). At present, the research of antitumor drugs is mostly concentrated in the field of apoptosis, but almost all tumor cells will appear to have apoptosis resistance. But luckily, some highly malignant cancer cells have been shown to be innately susceptible to ferroptosis even after apoptosis resistance. All PCa cells are sensitive to ferroptosis-inducers, erastin and RSL3, even among castration-resistant PCa (Ghoochani et al., 2021). Therefore, induction of ferroptosis has been thought to be a new cancer treatment method (Zou et al., 2019).

It has been proved that ferroptosis occurs among various cancers, including liver cancer, lung cancer, pancreatic cancer, cervical cancer, osteosarcoma, head and neck carcinoma, and ovarian cancer (Lachaier et al., 2014; Sun et al., 2015; Hayano et al., 2016; Lin et al., 2016; Alvarez et al., 2017; Basuli et al., 2017). However, there are few studies linking ferroptosis to the development of PCa. Therefore, we tried to establish a ferroptosis-related gene prognostic index (FGPI) to predict 
BCR after RRT, and to predict radiation resistance and to limn the tumor microenvironment of PCa recurrence.

\section{METHODS}

\section{Data Collection}

We merged GSE79021 (Sinnott et al., 2017), GSE32571 (Kuner et al., 2013), GSE62872 (Penney et al., 2015), and GSE116918 (Jain et al., 2018) from the Gene Expression Omnibus (GEO) database and removed batch effects (Edgar et al., 2002). We extracted the expression of messenger RNA (mRNA) and long noncoding RNA (lncRNA) from the combined datasets. The former three GEO datasets (Kuner et al., 2013; Penney et al., 2015; Sinnott et al., 2017) contained 248 normal and 476 tumor tissues which were used to identify the differentially expressed genes (DEGs) using the R package "limma" and the tumor-related genes by weighted gene co-expression network analysis (WGCNA). Tumor-related genes were defined as the absolute value of coefficient $\geq 0.3$ and p.adj. $<0.0001$, and DEGs were defined as the absolute value of $\log \mathrm{FC} \geq 0.4$ and p.adj. $<0.05$. Prognosis analysis was conducted using 248 tumor patients undergoing RRT with complete BCR data in GSE116918 (Jain et al., 2018). Besides, we obtained the PCa data in The Cancer Genome Atlas (TCGA) from UCSC XENA (Goldman et al., 2020) and downloaded ferroptosis-related genes from the Genecards database (Stelzer et al., 2016). By taking the intersection of tumor-related genes, DEGs, and ferroptosisrelated genes, we obtained the candidate genes. For PCa patients undergoing RRT in GSE116918 (Jain et al., 2018), ACSL3, EPAS1, and NEDD4L were identified through the Lasso regression analysis, and COX regression analysis was further performed using these three genes. Moreover, we observed that ACSL3 and EPAS1 were the independent gene factors of BCR through the COX multivariate analysis, and we subsequently constructed the FGPI based on the coefficients of the two genes. The FGPI $=-0.930^{\star}$ ACSL3-1.902 ${ }^{\star}$ PPAS1. In addition, we enrolled the FGPI and clinical features in GSE116918 (Jain et al., 2018) into the COX regression analysis and further confirmed that the FGPI was an independent prognostic factor for BCR. 70\% of patients from GSE116918 (Jain et al., 2018) were randomly extracted to internally verify the FGPI's prognostic value of BCR. In addition, $425 \mathrm{PCa}$ patients undergoing RP with complete prognostic data of metastasis in TCGA database were used to externally validate the prognostic value of the FGPI score. GSE53902 (Li et al., 2020) sequenced nine radio-resistant and nine normal DU145 cell lines, which were used to externally confirm the FGPI's prediction value of radiation resistance. Moreover, we analyzed the relationship between immune indicators and FGPI score.

\section{Gene Interaction Analysis and Function Enrichment Analysis}

We explored the protein-protein interaction of ACSL3 and EPAS1 through the GeneMANIA database (Warde-Farley et al., 2010). We identified lncRNAs which were differentially

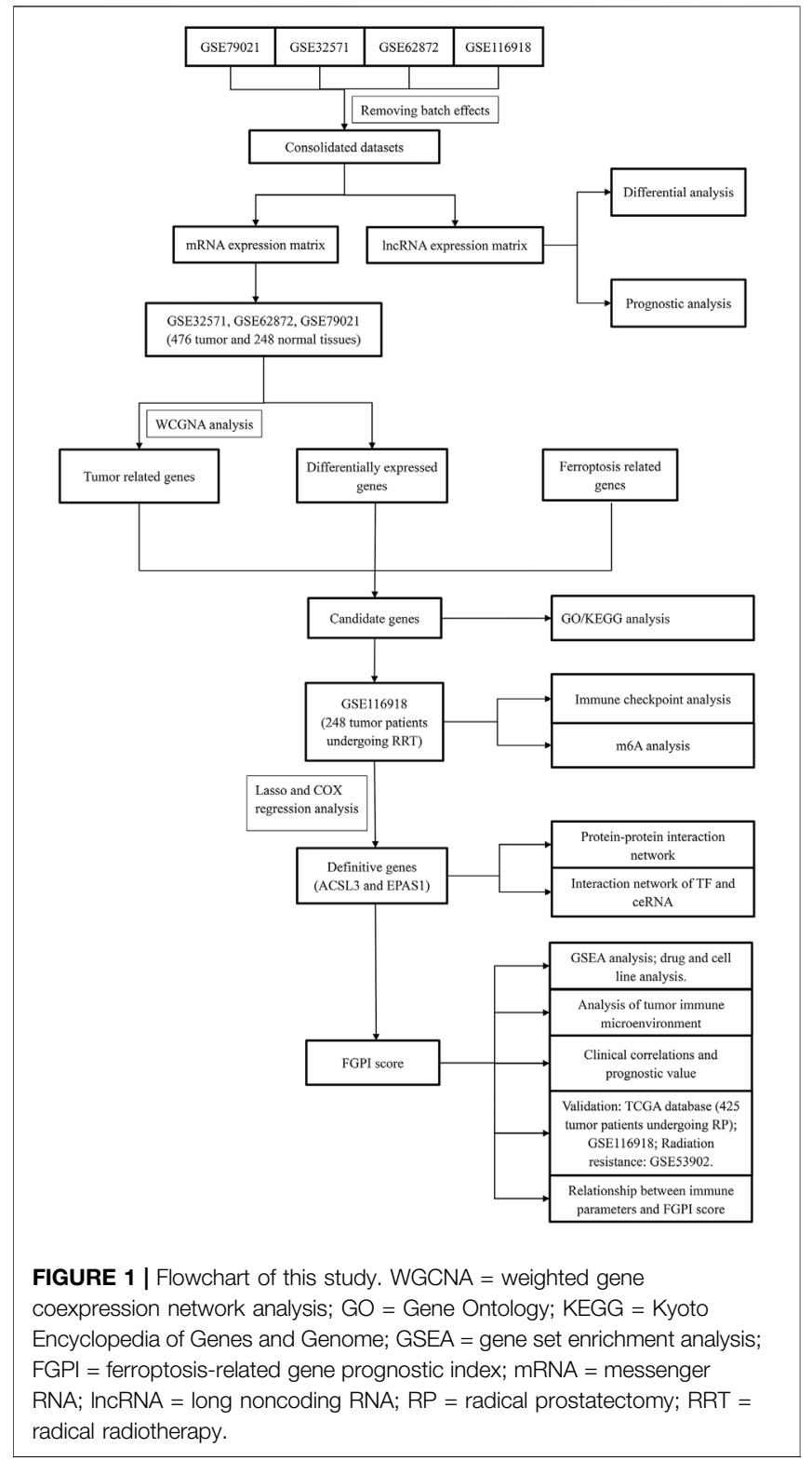

expressed between 248 normal and 476 tumor tissues in GSE79021 (Sinnott et al., 2017), GSE32571 (Kuner et al., 2013), and GSE62872 (Penney et al., 2015) and associated with BCR in GSE116918 (Jain et al., 2018). Subsequently, we established the interaction network of competing endogenous RNA (ceRNA) and transcription factor through lncBase (Paraskevopoulou et al., 2016), miWalk (Sticht et al., 2018), and TRRUST (Han et al., 2018). We analyzed the potential biological functions and signaling pathways through Gene Ontology (GO) and Kyoto Encyclopedia of Genes and Genome (KEGG) analysis of candidate genes. GO analysis consisted of biological process, cell composition, and molecular function. We divided the PCa patients undergoing RRT from GSE116918 (Jain et al., 2018) into high- and low-risk groups according to the median of the FGPI score. Gene set enrichment analysis (GSEA) of high- and low-risk groups from 


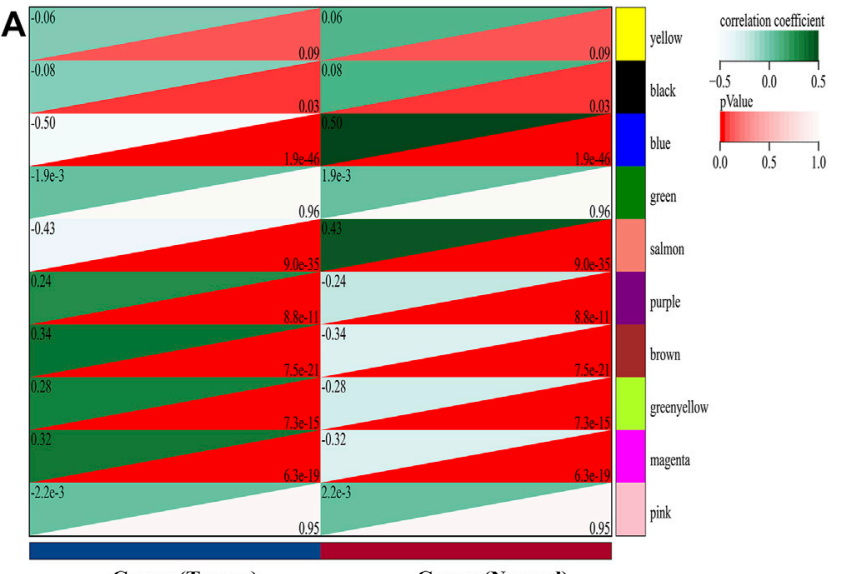

Group (Tumor)

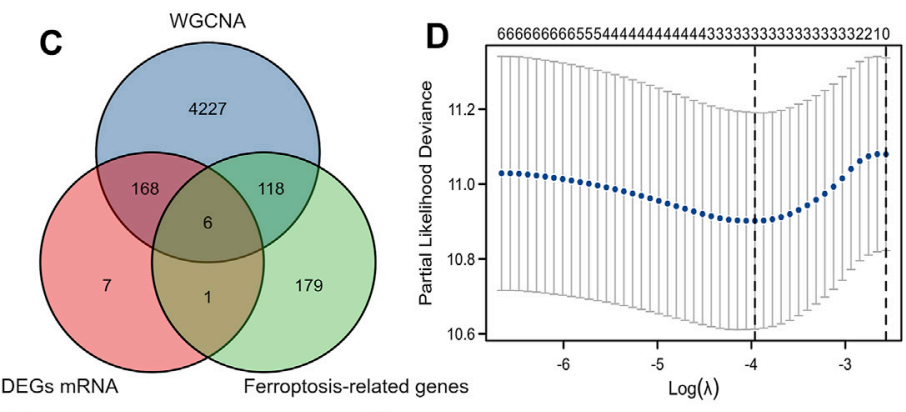

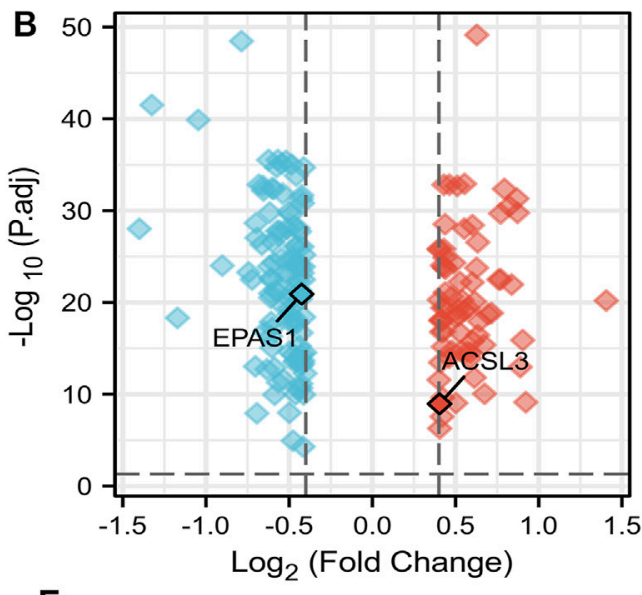

E

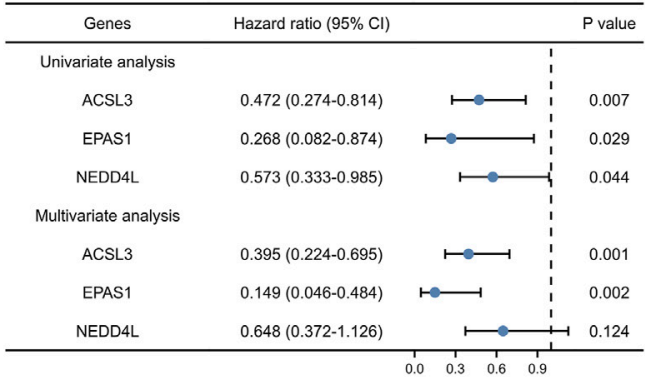

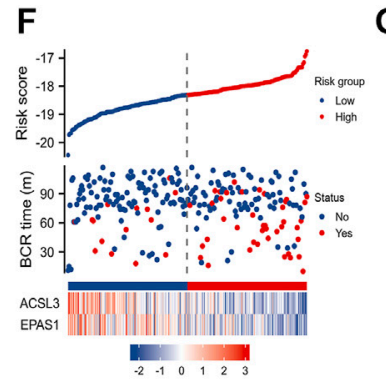
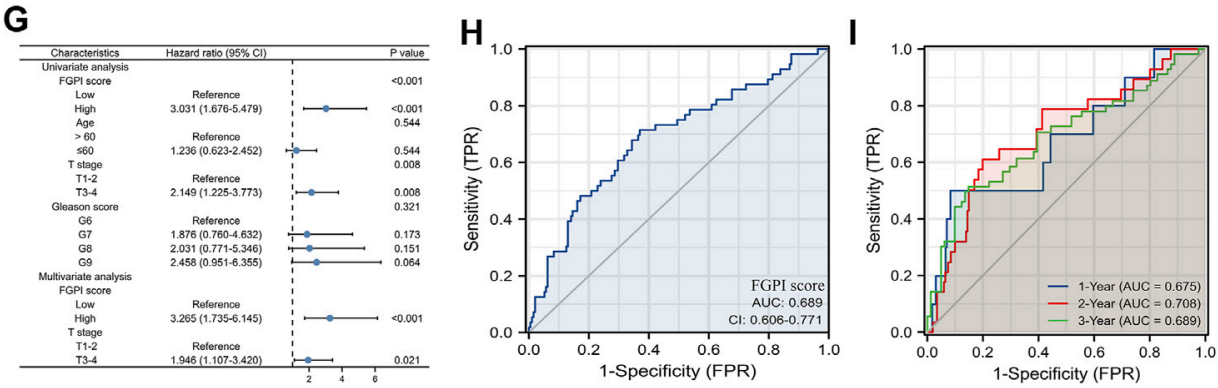

FIGURE 2 | Identification of the FGPI score. (A) Modules and phenotype showing tumor-related genes using blue, salmon, brown, and magenta modules; (B) volcano plot presenting the results of DEGs between 248 normal and 476 tumor tissues in the three GEO datasets (Kuner et al., 2013; Penney et al., 2015; Sinnott et al., 2017); (C) Venn diagram showing the results of the intersection of tumor-related genes, DEGs, and ferroptosis-related genes; (D) identifying three genes (ACSL3, EPAS1, and NEDD4L) using the Lasso regression analysis; (E) identifying genes independently associated with BCR in GSE116918 (Jain et al., 2018) using the COX regression; (F) risk factor plot showing the prognostic data and mRNA expression of ACSL3 and EPAS1; (G) determining the prognostic value of the FGPI score for BCR-free survival through the univariate and multivariate COX analyses including the clinical features of PCa patients undergoing RRT in GSE116918 (Jain et al., 2018); (H) ROC curve discriminating BCR from no BCR for PCa patients undergoing RRT in GSE116918 (Jain et al., 2018) using the FGPI score; (I) time-dependent ROC curve discriminating BCR from no BCR for PCa patients undergoing RRT in GSE116918 (Jain et al., 2018) using the FGPI score. FGPI = ferroptosis-related gene prognostic index; $\mathrm{ROC}=$ receiver operating characteristic; $\mathrm{BCR}=$ biochemical recurrence; GEO = Gene Expression Omnibus.

GSE116918 (Jain et al., 2018) was conducted as well. "c2.cp.kegg.v7.4.symbols.gmt" and "h.all.v7.4.symbols.gmt" were downloaded from the molecular signature database to evaluate related pathways and molecular mechanisms (Liberzon et al., 2011). P. adj. $<0.05$ and false discovery rate $<0.25$ were considered statistically significant.

\section{Drug Analysis and TME Analysis}

We analyzed the drug sensitivity of ACSL3 and EPAS1 through GSCALite which conducted drug analysis from the Cancer Therapeutics Response Portal (CTRP) and genomics of drug sensitivity in cancer (GDSC) (Liu et al., 2018). We analyzed the differential expression of the 17 common immune checkpoints in BCR and no BCR groups. EPIC (Racle et al., 2017), ESTIMATE (Yoshihara et al., 2013), and immunophenoscore (IPS) (Charoentong et al., 2017) algorithms were used to assess the TME of GSE116918 (Jain et al., 2018) through the R package "IOBR" (Zeng et al., 2021). Similarly, differential expression of TME-related cells in BCR and no BCR groups and their relationship with FGPI score were analyzed as well. We presented the flowchart of this study in Figure 1. 
A
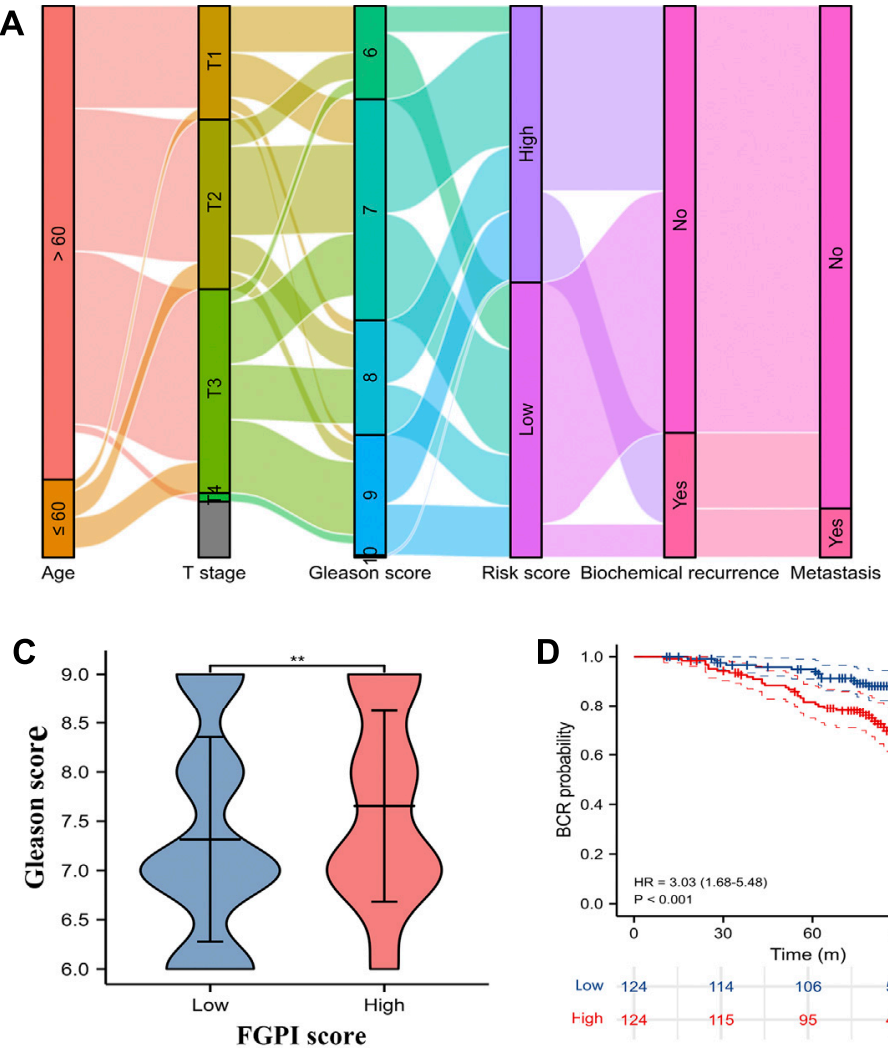

F

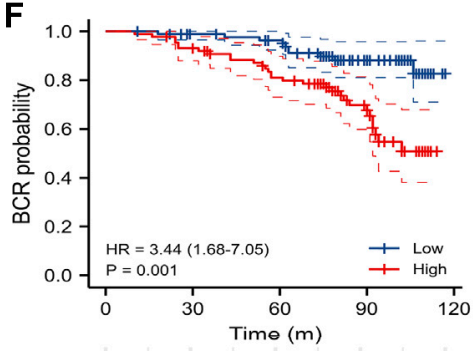

\begin{tabular}{l|l|l|l|l|l|} 
Low & 87 & 81 & 76 & 36 & 0
\end{tabular}

$\begin{array}{lllllll}\text { High } & 88 & 81 & 66 & 33 & 0\end{array}$

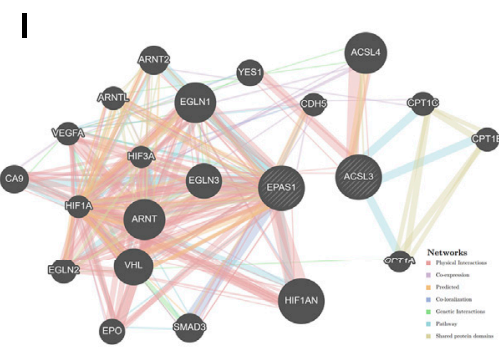

J

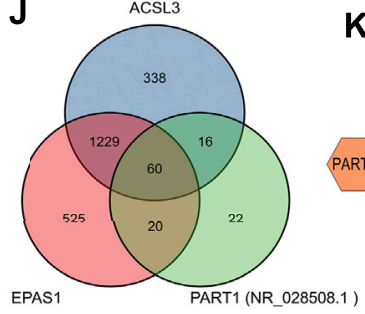

D

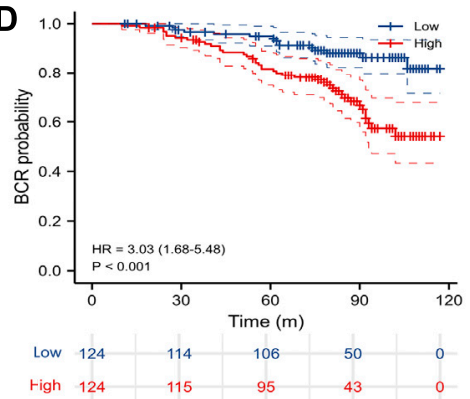

G

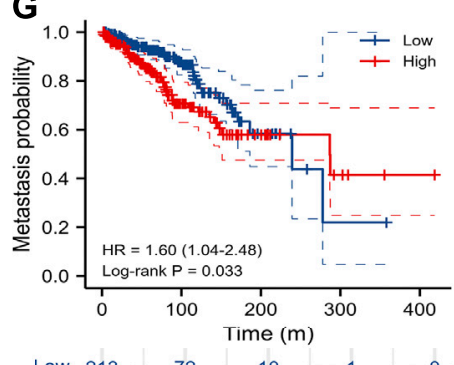

\begin{tabular}{l|l|l|l|l|l} 
Low & 213 & 72 & 10 & 1 & 0
\end{tabular}

\begin{tabular}{ll|l|l|l|l} 
High & 213 & 50 & 13 & 4 & 1
\end{tabular}

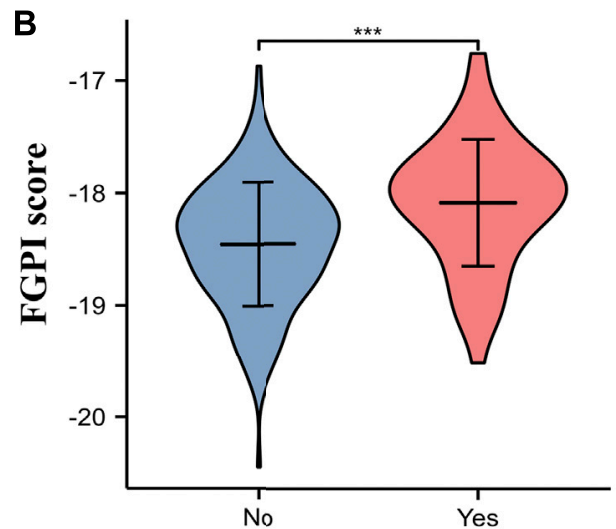

Biochemical recurrence

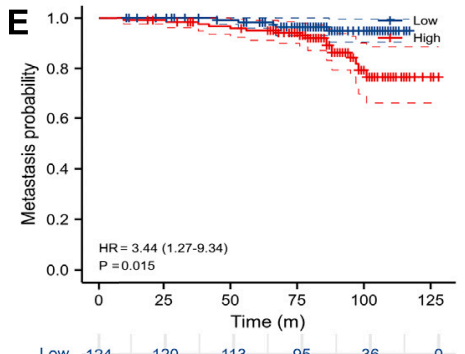

\begin{tabular}{ll|l|l|l|l|l} 
Low 124 & 120 & 113 & 95 & 36 & 0
\end{tabular}

\begin{tabular}{l|l|l|l|l|l|l} 
High & 124 & 121 & 114 & 97 & 30 & 3
\end{tabular}

H

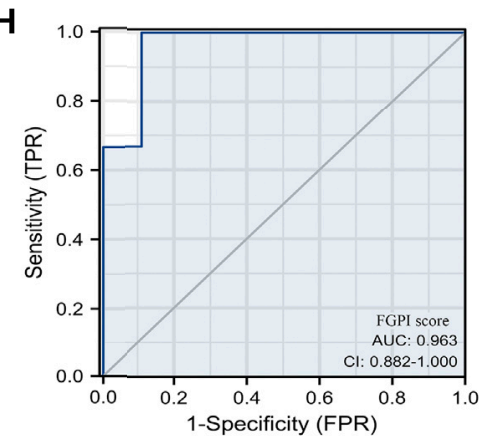

K

FIGURE 3 | Clinical values and interaction network. (A) Sankey plot showing flow trend of clinical data and outcomes in GSE116918 (Jain et al., 2018); (B) comparison of FGPI score between BCR and no BCR groups for PCa patients in GSE116918 (Jain et al., 2018); (C) PCa patients in GSE116918 (Jain et al., 2018) were divided into high- and low-risk groups based on the median of the FGPI score and comparison of Gleason score between high- and low-risk groups; (D) Kaplan-Meier curve showing the difference of BCR-free survival for PCa patients undergoing RRT in GSE116918 (Jain et al., 2018 ) according to the median of the FGPI score; (E) Kaplan-Meier curve showing the difference of metastasis-free survival for PCa patients undergoing RRT in GSE116918 (Jain et al., 2018) according to the median of the FGPI score; (F) Kaplan-Meier curve of the internal validation showing the difference of BCR-free survival for $70 \%$ of PCa patients undergoing RRT in the GSE116918 (Jain et al., 2018) according to the median of the FGPI score; (G) Kaplan-Meier curve showing the difference of metastasis-free survival for PCa patients undergoing RP in TCGA database according to the median of the FGPI score; (H) ROC curve showing the diagnostic accuracy of the 
FIGURE 3 | FGPI score for radioresistance; (I) protein-protein interaction network showing genes might interact with ACSL3 and EPAS1 using the GeneMANIA database (Warde-Farley et al., 2010); (J) Venn plot showing potentially interacting miRNAs of ACSL3, EPAS1, and PART1; (K) interaction network of competing endogenous RNAs and transcription factors. ROC = receiver operating characteristic; FGPI = ferroptosis-related gene prognostic index; BCR = biochemical recurrence; $\mathrm{RRT}$ = radical radiotherapy; $\mathrm{RP}$ = radical prostatectomy.

\section{Statistical Analysis}

All analyses were conducted with $\mathrm{R}$ version 3.6.3 and its suitable packages. Cytoscape 3.8.2 was used to establish ceRNA and transcription factor network. BCR-free survival was the primary outcome, and metastasis-free survival (MFS) was the secondary outcome. The Wilcoxon test was used if data distribution does not satisfy normality. Only variables that are statistically significant in the univariable Cox regression analysis were included in the multivariable Cox regression models. Each outcome was regarded as statistically significant with two-sided $p$-value $<0.05$. Significant mark: ns, $p \geq 0.05$; ${ }^{*}, p<0.05$; ${ }^{* *}, p<$ $0.01 ;{ }^{* * *}$, and $p<0.001$.

\section{RESULTS}

\section{Data Presentation and Clinical Value}

We merged the four GEO datasets by removing batch effects (Supplementary Figure S1) (Kuner et al., 2013; Penney et al., 2015; Sinnott et al., 2017; Jain et al., 2018). We used the WGCNA analysis to identify the tumor-related genes through the three GEO datasets (Kuner et al., 2013; Penney et al., 2015; Sinnott et al., 2017). The blue, salmon, brown, and magenta modules contained 4519 genes associated with the tumor according to the abovementioned definition (Figure 2A). We used the volcano plot to present the results of DEGs between 248 normal and 476 tumor tissues in the three GEO datasets (Kuner et al., 2013; Penney et al., 2015; Sinnott et al., 2017). ACSL3, EPAS1, FASN, GSTP1, LDHB, and NEDD4L were identified as the candidate genes through the intersection of tumor-related genes, DEGs, and ferroptosis-related genes (Figure 2C). We enrolled ACSL3, EPAS1, and NEDD4L identified from the Lasso regression analysis (Figure 2D) into the COX regression analysis to identify the genes which were independent risk factors of BCR for patients undergoing RRT in GSE116918 (Jain et al., 2018) (Figure 2E). The risk factor diagram shows the difference in the distribution of the two gene expressions among the high- and low-risk groups (Figure 2F). We observed that FGPI score was an independent risk factor of $\mathrm{PCa}$ patients through COX multivariate analysis (HR: 3.265, 95\% CI: 1.735-6.145; Figure 2G). The ROC curve showed that our FGPI score had low diagnostic accuracy (AUC: 0.689; Figure 2H) in discriminating BCR from no BCR within 1, 2, and 3 years (Figure 2I).

The flow trend of clinical data and outcomes in GSE116918 (Jain et al., 2018) was presented. (Figure 3A). We observed weak correlation between FGPI score and age ( $\mathrm{r}: 0.130, p=0.048)$. For PCa patients undergoing RRT in GSE116918 (Jain et al., 2018), BCR patients had a significantly higher FGPI score than no BCR patients (Figure 3B), and patients in the high-risk group had a significantly higher Gleason score than those in the low-risk group (Figure 3C). The Kaplan-Meier curve showed that higher FGPI score was an independent risk factor for BCR (HR: 3.03, 95\% CI: 1.68-5.48; $p<0.001$; Figure 3D) and MFS (HR: 3.44, 95\% CI: $1.27-9.34, p=0.015$; Figure 3E) for PCa patients undergoing RRT in GSE116918 (Jain et al., 2018). In internal certification using the patients from GSE1 16918 (Jain et al., 2018), we observed a similar result (HR: 3.44; 95\% CI: $1.68-7.05$; $p=$ 0.001; Figure 3F). For PCa patients undergoing RP in TCGA database, we detected that patients in the high-risk group were at higher risk of metastasis than those in the low-risk group (HR: 1.60, 95\% CI: 1.04-2.48; Figure 3G). The ROC curve showed a strong diagnostic value of FGPI for the radiation resistance of PCa (AUC: 0.963, 95\% CI: 0.882-1.000; Figure 3H). Through the GeneMANIA database (Warde-Farley et al., 2010), we found that ACSL3 and EPAS1 might work together through coexpression and other genes (Figure 3I). In the ceRNA network, the lnRNA PART1 might modulate the expression of ACSL3 and EPAS1 through 60 miRNAs (Figures 3J,K). RELA could activate the expression of EPAS1, while YY1 could suppress the expression of EPAS1 (Figure 3K).

\section{Function Enrichment Analysis}

GO and KEGG analyses indicated that the candidate genes are mainly engaged in the fatty-acyl-CoA metabolic process, nucleoside and ribonucleoside bisphosphate biosynthetic process, regulation of lipid biosynthetic process, cellular response to oxidative stress, and fatty acid biosynthesis and metabolism, as well as GSH binding, coenzyme binding, and fatty acid ligase activity (Figures 4A,B). GSEA analysis showed that high-risk patients were enriched in epithelial-mesenchymal transition, allograft rejection, Fc gamma R-mediated phagocytosis, TGF beta signaling pathway, and ECM receptor interaction, while low-risk patients were enriched in adipocytokine signaling pathway androgen response and notch signaling (Figures 4C,D).

\section{Drug and Immunologic Analysis}

We presented the top 30 correlations between CTRP drug sensitivity and mRNA expression of ACSL3 and EPAS1 in pan cancer (Figure 5A), as well as the correlation between GDSC drug sensitivity and mRNA expression of ACSL3 and EPAS1 in pan cancer (Figure 5B). We identified nine potentially sensitive drugs through intersection of GDSC-EPAS1, GDSC-ACSL3, CTRPACSL31, and CTRP-EPAS1 (Figure 5C). The nine possible drugs were OSI-027, OSI-930, PAC-1, PHA-793887, PI-103, PIK-93, SNX-2112, TPCA-1, and UNC0638. m6A analysis showed that METTL14 was expressed significantly lower in the BCR group than the no BCR group (Figure 5D). Immune checkpoint analysis showed that mRNA expression of PDCD1LG2 (PD-L2) and CD96 was significantly higher in the $\mathrm{BCR}$ group than that of no BCR groups (Figures 5D,E). We 
A
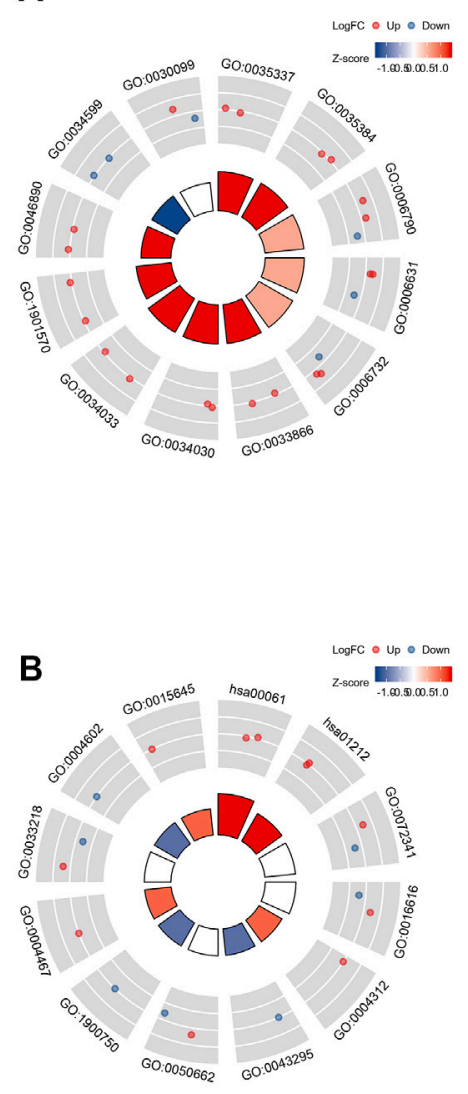

C

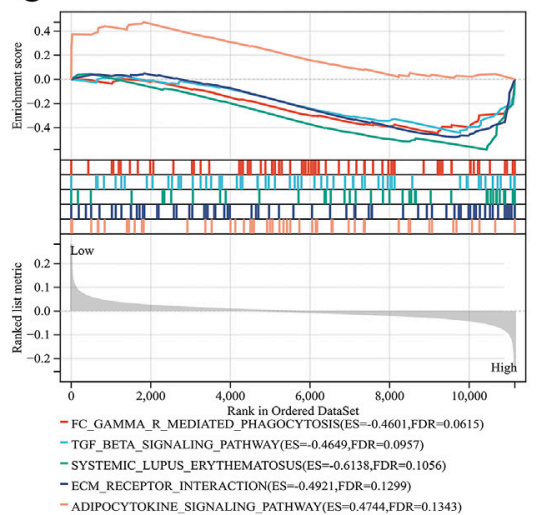

\begin{tabular}{|c|c|c|c|c|c|c|}
\hline Ontology & ID & Description & Generatio & BgRatio & p.adjust & qualue \\
\hline $\mathrm{BP}$ & GO:0035337 & $\begin{array}{c}\text { fatty-acyl-CoA } \\
\text { metabolic process }\end{array}$ & $2 / 6$ & $41 / 118670$ & 0.008 & 0.003 \\
\hline BP & GO:0035384 & $\begin{array}{c}\text { thicester biosynthetic } \\
\text { process }\end{array}$ & $2 / 6$ & $54 / 18670$ & 0.008 & 0.003 \\
\hline BP & GO 0006790 & $\begin{array}{l}\text { sulfur compound } \\
\text { metabolic process }\end{array}$ & $3 / 6$ & $372 / 18670$ & 0.008 & 0.003 \\
\hline $\mathrm{BP}$ & GO:0006631 & $\begin{array}{l}\text { fatty acid metabolic } \\
\text { process }\end{array}$ & $3 / 6$ & $383 / 18670$ & 0.008 & 0.003 \\
\hline BP & GO:0006732 & $\begin{array}{c}\text { coenzyme metabolic } \\
\text { process }\end{array}$ & $3 / 6$ & $403 / 18670$ & 0.008 & 0.003 \\
\hline BP & G0:0033866 & $\begin{array}{c}\text { mucleoside } \\
\text { bisphoshhate } \\
\text { biosynhhtic arocess }\end{array}$ & $2 / 6$ & $68 / 18670$ & 0.008 & 0.003 \\
\hline BP & GO:0034030 & $\begin{array}{c}\text { ribonucleoside } \\
\text { bisphosphate } \\
\text { biosynhhtic process }\end{array}$ & $2 / 6$ & $68 / 18670$ & 0.008 & 0.003 \\
\hline BP & GO:0034033 & $\begin{array}{c}\text { purine nucleoside } \\
\text { bisphosphate } \\
\text { biosyntheic process }\end{array}$ & $2 / 6$ & $68 / 18670$ & 0.008 & 0.003 \\
\hline BP & GO: 1901570 & $\begin{array}{l}\text { fatty acid derivative } \\
\text { biosynthetic process }\end{array}$ & $2 / 6$ & 99/18670 & 0.014 & 0.007 \\
\hline $\mathrm{BP}$ & GO:0046890 & $\begin{array}{l}\text { regulation of lipid } \\
\text { biosynthctic process }\end{array}$ & $2 / 6$ & $198 / 18670$ & 0.034 & 0.015 \\
\hline BP & GO:0034599 & $\begin{array}{l}\text { cellular response to } \\
\text { oxidative stress }\end{array}$ & $2 / 6$ & $302 / 18670$ & 0.037 & 0.017 \\
\hline $\mathrm{BP}$ & GO:0030099 & $\begin{array}{l}\text { mycloid oell } \\
\text { differentiation }\end{array}$ & $2 / 6$ & $416 / 18670$ & 0.043 & 0.019 \\
\hline Ontology & ID & Description & GeneRatio & BgRatio & p.adjust & qvalue \\
\hline MF & GO:0072341 & $\begin{array}{l}\text { modified amino acid } \\
\text { binding }\end{array}$ & $2 / 6$ & 89/17697 & 0.013 & 0.003 \\
\hline MF & GO:0016616 & $\begin{array}{l}\text { oxidoreductase activity, } \\
\text { acting on the CH-OH } \\
\text { group of donors, NAD or } \\
\text { NADP as acceptor }\end{array}$ & 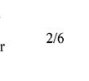 & $119 / 17697$ & 0.013 & 0.003 \\
\hline MF & GO:0004312 & $\begin{array}{l}\text { fatty acid synthase } \\
\text { activity }\end{array}$ & $1 / 6$ & $11 / 17697$ & 0.028 & 0.007 \\
\hline MF & GO:0043295 & glutathione binding & $1 / 6$ & $11 / 17697$ & 0.028 & 0.007 \\
\hline MF & GO:0050662 & coenzyme binding & $2 / 6$ & $291 / 17697$ & 0.028 & 0.007 \\
\hline MF & GO:1900750 & oligopeptide binding & $1 / 6$ & $12 / 17697$ & 0.028 & 0.007 \\
\hline MF & GO:0004467 & $\begin{array}{l}\text { long-chain fatty acid-CoA } \\
\text { ligase activity }\end{array}$ & $1 / 6$ & $13 / 17697$ & 0.028 & 0.007 \\
\hline MF & GO:0033218 & amide binding & $2 / 6$ & $356 / 17697$ & 0.028 & 0.007 \\
\hline MF & GO:0004602 & $\begin{array}{l}\text { glutathione peroxidase } \\
\text { activity }\end{array}$ & $1 / 6$ & $21 / 17697$ & 0.029 & 0.007 \\
\hline MF & GO:0015645 & fatty acid ligase activity & $1 / 6$ & $21 / 17697$ & 0.029 & 0.007 \\
\hline KEGG & hsa00061 & Fatty acid biosynthesis & $2 / 6$ & $18 / 8076$ & 0.002 & 0.002 \\
\hline KEGG & hsa01212 & Fatty acid metabolism & 2/6 & $57 / 8076$ & 0.011 & 0.008 \\
\hline
\end{tabular}

D

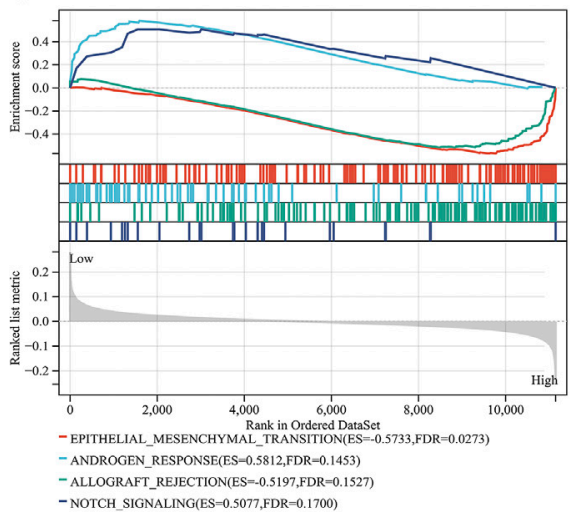

FIGURE 4 | Functional enrichment analysis. (A) GO analysis showing the results of BP; (B) GO analysis showing the results of MF and KEGG analysis; (C) GSEA analysis of high- and low-risk groups (according to the median of the FPGl score) for PCa patients undergoing RRT in GSE116918 (Jain et al., 2018 ) using the subset of "c2.cp.kegg.v7.4.symbols.gmt;" (D) GSEA analysis of high- and low-risk groups (according to the median of the FPGl score) for PCa patients undergoing RRT in GSE116918 (Jain et al., 2018) using the subset of "h.all.v7.4.symbols.gmt." GO = Gene Ontology; KEGG = Kyoto Encyclopedia of Genes and Genome; GSEA = gene set enrichment analysis; $\mathrm{BP}=$ biological process; $\mathrm{MF}=$ molecular function.

divided the 248 PCa patients from GSE116918 (Jain et al., 2018) into high- and low-risk groups according to the median of the expression of PD-L2 or CD96. Through the survival analysis curve, we found that only CD96 was significantly associated with BCR-free survival (HR: 1.79; 95\% CI: 1.06-3.03; Figure 5F). For
TME analysis, we observed that between $\mathrm{BCR}$ and no $\mathrm{BCR}$ groups, cancer-related fibroblasts, macrophages, stromal score, immune score, estimate score, and tumor purity were differentially expressed and were risk factors for BCR (HRs were $2.17,1.79,2.20,1.93,1.92$, and 0.52 for cancer-related 


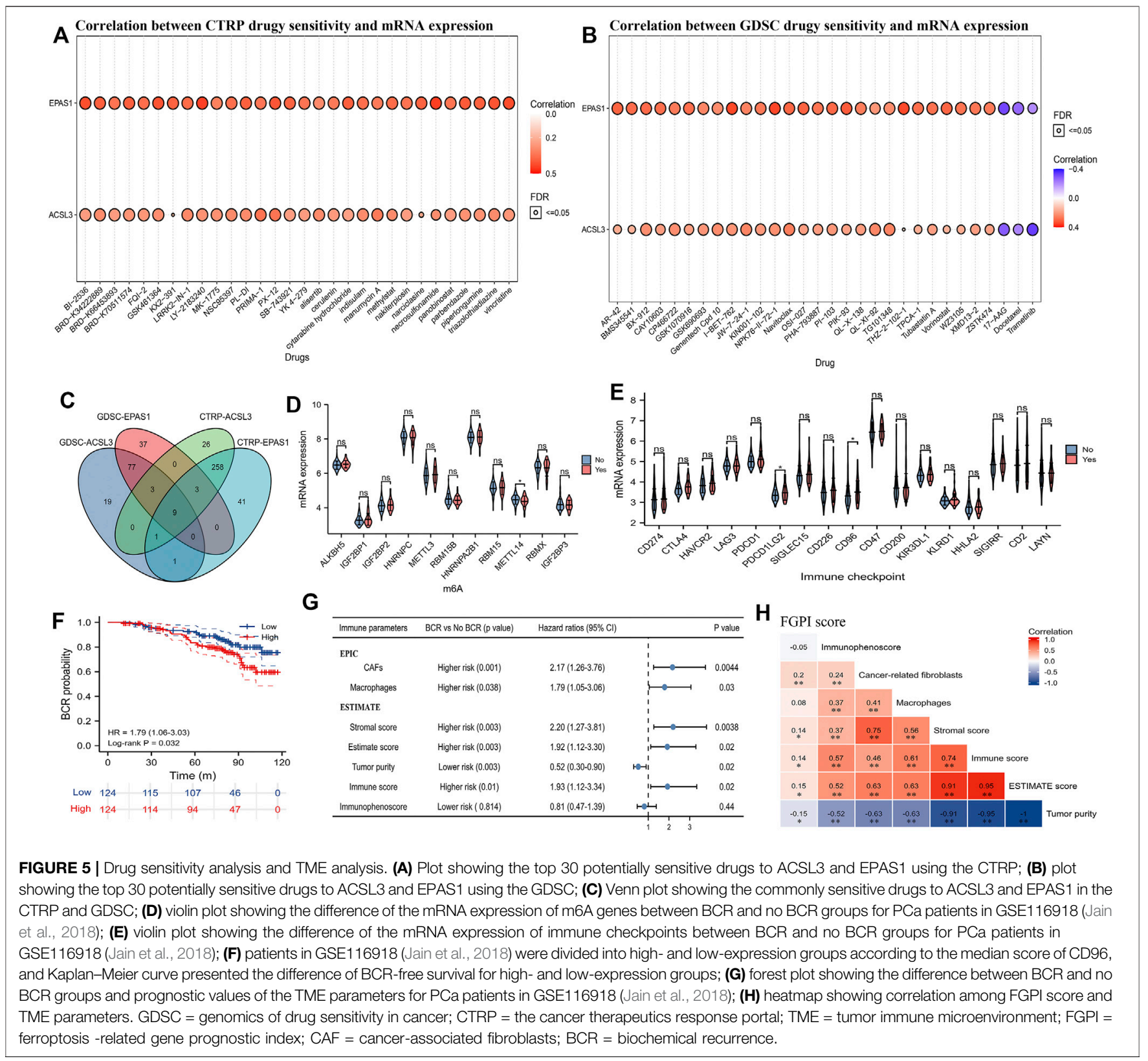

fibroblasts, macrophages, stromal score, immune score, estimate score, and tumor purity, respectively; Figure 5G). Moreover, cancer-related fibroblasts (coefficient: 0.20), stromal score (coefficient: 0.14), immune score (coefficient: 0.14), estimate score (coefficient: 0.15 ), and tumor purity (coefficient: -0.15 ) were significantly related to FGPI (Figure 5H).

\section{DISCUSSION}

Compared with other cancers, PCa exhibits very special metabolic characteristics, such as early reliance on mitochondrial metabolism and dysregulation of both fatty acid synthesis and oxidation pathways, which may endow PCa cells with the characteristics of ferroptosis tendency. The 10-year overall survival and cancer specific survival of RRT was comparable to RP in prospective trials (Wallis et al., 2016; Wallis et al., 2018). And, salvage radiotherapy (SRT) is the first choice after BCR (Boorjian et al., 2009; Wiegel et al., 2009). The main challenge of PCa management is not the lack of initial treatment options, but treatment adaptation leading to resistance. It is generally agreed that the goal of defining BCR is to determine an early sign of treatment failure before clinical treatment. In the era of precision medicine, it is a new method to predict BCR based on individual tumor-related genes for selecting RRT indications. However, there is a lack of effective prediction and management measures for the problems of RRT resistance and BCR. 
Ferroptosis is an attractive and very promising concept that can combat the resistance of various types of cancer to multiple therapies. Ferroptosis is considered a natural barrier to the progression of various cancers (Xu et al., 2019; Bebber et al., 2020). Recent research has focused more on the application of ferroptosis inducers (such as erastin) to treat drug-resistant castration-resistant prostate cancer (CRPC) (Yi et al., 2020; Ghoochani et al., 2021). However, there are few studies linking ferroptosis to $\mathrm{BCR}$ and radiation resistance among $\mathrm{PCa}$. Previous studies have found that the overexpression of Tip60, LDH5, AKR1C3, miR-191, etc. confers PCa resistance to radiotherapy (Koukourakis et al., 2014; Sun et al., 2016; Xie et al., 2018). In this article, we first determined an FGPI comprising ferroptosisrelated genes, ACSL3 and EPAS1, which has valuable and independent significance for predicting both BCR and radiation resistance. ACSL3 has been found to contribute to the growth of PCa in the mouse model, and high expression of ACSL3 is associated with poor prognosis (Obinata et al., 2016). Furthermore, some researchers have discovered that ACSL3 can mediate the activation of monounsaturated fatty acids and promote the function of oleic acid, thereby potently inhibiting ferroptosis of tumor cells (Magtanong et al., 2019; Ubellacker et al., 2020). Interestingly, in ovarian cancer, however, the homozygous deletion of ACSL3 is significantly related to the increased risk of recurrence in patients treated with adjuvant chemotherapy, suggesting that ACSL3 may be cancer-specific and possess complicate functions (Jeong et al., 2017). EPAS1 is a hypoxia-inducible gene encoding hypoxia-inducible factor $2 \alpha$ (HIF-2 $\alpha$ ). Research by Peng et al. showed that EPAS1 siRNA nanoparticles can inhibit the proliferation of pancreatic cancer cells and induce apoptosis under hypoxic conditions (Peng et al., 2017). EPAS1/HIF2a may be a key transcriptional control target for tumor cells to respond to hypoxia, which is a characteristic of aggressive tumor cells, and is also involved in resistance to chemotherapy and ionizing radiation (Peng et al., 2017; Påhlman and Mohlin, 2018).

In this study, we observed that the IncRNA PART1 mediated the expression of EPAS1 and ACSL3 and was significantly associated with BCR in PCa. Multiple studies have found that the overexpression of the IncRNA PART1 promoted the infiltration of mononuclear immune cells, prediction to current immune checkpoint gene markers, inhibition of tumor proliferation, promotion of cell apoptosis, and suppression of cell invasion in bladder cancer (Hu et al., 2019). At the same time, the lncRNA PART1 is also involved in the progression of renal clear cell carcinoma (Liu et al., 2019; Zhou et al., 2021). The epithelial-mesenchymal transition (EMT) plays an important role in the progression of $\mathrm{PCa}$, characterized by morphological changes in their phenotype from cuboidal to spindle-shaped (Montanari et al., 2017; Odero-Marah et al., 2018). Research by Yan et al. showed that in the EMT model of PCa, circRNAs regulate EMT through $\mathrm{PI} 3 \mathrm{~K}-\mathrm{Akt}$ signaling and TGF beta signaling pathways (Yan et al., 2020). Meanwhile, the oncogenic activation of the PI3K-AKT-mTOR signaling pathway has been shown to be related to the inhibition of ferroptosis via the sterol regulatory element-binding proteinmediated lipogenesis (Yi et al., 2020). Now, we speculated that based on our results, the interaction of the lncRNAs PART1, EPAS1, and ACSL3 may promote EMT through PI3K-Akt signaling and TGF- $\beta$ signaling pathways, thereby causing the occurrence of BCR in PCa, with ferroptosis as one of the important links in this process. The complicated mechanism requires further study.

CD96, which is mainly expressed in natural killer (NK) cells and $\mathrm{T}$ cells, acts as a vital checkpoint in immunity and tumor progression, with CD96 mice displaying hypersensitive NK-cell responses to immune challenge and significant tumor resistance (Blake et al., 2016). By blocking CD96 in mice, the growth of primary tumors was inhibited, thus promoting greater tumor control (Mittal et al., 2019). CD96 has already been clearly associated with the prognosis of human hepatocellular carcinoma, advanced ovarian cancer, and glioma (Sun et al., 2019; Liu et al., 2020; Maas et al., 2020; Zhang et al., 2020). In pancancer analysis, CD96 was found to affect immune cell infiltration and malignant properties, thereby significantly affecting the prognosis of various cancers (Ye et al., 2021). This antitumor immune function of CD96 may be derived from the interaction of CD155/CD112 on tumor cells or antigen-presenting cells in the TME with TIGIT/CD96 on CD8 T cells or NK cells, resulting in the inhibition of antitumor NK cells and T cell functions (Dougall et al., 2017). The study by Du et al. showed that in PCa, CD96 can affect the late phase of immune response and $\mathrm{PCa}$ development through physical interaction with the PCa risk locus 8q24 (Du et al., 2015). In our research, CD96 was first found to be related to the time when BCR occurred in PCa. We speculate that CD96 may weaken the antitumor immune function of NK cells and CD8 $\mathrm{T}$ cells in the TME, achieve the purpose of immune evasion, make BCR occur early, and seriously affect the prognosis of patients, which makes CD96 a potential curative and preventive target for BCR. Another immune checkpoint, PD-L2, is closely related to immune-related pathways and radiation response pathway, and also possesses the ability to predict the response to postoperative radiation therapy (Zhao et al., 2019). Combined with our research, we speculate that PDL2 is also one of the immune checkpoints and indicators for evaluating prognosis and resistance for $\mathrm{PCa}$.

The TME of PCa is a complex cancer-promoting and suppressing environment, which includes cancer cells and non-cancer cells, such as fibroblasts, immune cells, endothelial cells, and normal epithelial cells (Joyce and Pollard, 2009). This mixture is believed to play an important role in tumor progression and resistance (Hanahan and Weinberg, 2011; Junttila and de Sauvage, 2013). PCa is generally defined as "cold tumor," which means the immune cells in the TME are lesser (Danaher et al., 2018). Our research shows that the higher immune score, the lower the purity of the tumor, the greater the probability of BCR, and the lower the BCR-free survival. This may partly be attributed to the immune evasion mechanism mentioned above. Meanwhile, the inflammatory microenvironment, characterized by a high immune score and low tumor purity, will increase the mutation rate, and with the recruited white blood cells and lymphocytes expressing cytokines and inflammatory factors that promote tumor progression and induce angiogenesis and hypoxia, a tumor-friendly 
microenvironment is established (Soucek et al., 2007; Mantovani et al., 2008; Grivennikov et al., 2010; Aran et al., 2015). For example, the tumor-associated macrophages (TAMs) in the TME are the main source of cytokine secretion, among which M1 macrophages express high levels of pro-inflammatory factors TNF-a, IL-1, IL-6, IL-12, or IL -23, along with the M2 phenotype, as the main body of TAMs, expressing antiinflammatory cytokine IL-10, scavenger receptor A, and arginase, thus promoting tumor angiogenesis, invasion, metastasis, and tissue remodeling (Condeelis and Pollard, 2006; Mantovani et al., 2008; Sica et al., 2008). Moreover, in cancer stroma, CAFs, as the main component, can induce a cancerous phenotype and support tumor epithelial growth, invasion, and therapeutic resistance (Tripathi et al., 2012). The previous study showed that high density of CAFs might be associated with advanced-stage disease, higher Gleason scores, lymphatic metastases, higher PSA, and poor BCR-free survival in PCa patients (Wu et al., 2021). Besides, prostatic CAFs could induce tumorigenesis in normal human prostatic epithelial cells in vitro via the secretion of CXCL12, and this mechanism was found to be dependent on the presence of TGF- $\beta$ in vivo in a mouse model (Fiard et al., 2021). Consistent with the previous studies, in this study, we found that CAFs were highly associated with the poor BCR-free survival of PCa patients and TGF- $\beta$ was also highly enriched in the high-risk group. Combined with our research results, it is reasonable to believe that non-tumor cells in the TME, especially macrophages and fibroblasts, serve as seeds (cancer cells) in the soil (TME) and interact through stromal-epithelial interactions, which can promote the colonization of tumor cells and occurrence of PCa resistance (Hart and Fidler, 1980; Paget, 1989). The development of treatments that target TAMs and CAFs to prevent the growth of seeds in situ and its colonization at secondary sites is the focus of treatment goals. At the same time, the immune-related, genebased FGPI we developed can well reflect the characteristics of the immune microenvironment and predict BCR and MFS.

The previous study explored the role of ferroptosis-related genes in predicting BCR for PCa patients undergoing RP in TCGA database (Lv et al., 2021). They enrolled nine ferroptosisrelated genes (AIFM2, AKR1C1, AKR1C2, CBS, FANCD2, FTH1, G6PD, NFS1, and SLC1A5) into the model. In this study, we identified two different genes (ACSL3 and EPAS1) to predict the BCR-free survival for PCa patients undergoing RRT. We thought that the different sequencing methods, treatments, and data proceedings contributed to different conclusions. For the first time, we identified genes related to BCR and radiation resistance of iron death in PCa patients undergoing RRT and established a predicted FGPI. Our results suggest that ACSL3 and EPAS1 genes and related pathways are a method of iron death parallel to apoptosis, which is a potential therapeutic target for the treatment of drug-resistant $\mathrm{PCa}$, and also an indicator for predicting the occurrence of BCR. CD96 and PD-L2 are the two immune checkpoints of $\mathrm{PCa}$, and drugs targeting these two cell surface molecules may be effective in preventing BCR. Finally, the tumor microenvironment of PCa is also one of the research focuses of BCR in PCa. Despite these promising results, we have the following limitations. First, gene expression signatures are subject to sampling bias caused by intratumor genetic heterogeneity. Second, the microenvironment features might be distinct in different tumor regions, such as tumor core and invasive margin. More importantly, all findings, such as the ceRNA network and radioresistance in this study, still warranted to be further confirmed.

\section{CONCLUSION}

We found that FGPI based on ACSL3 and EPAS1 might be used to predict $\mathrm{BCR}$ and radiation resistance for PCa patients. CD96 and PD-L2 might be a possible target for drug action. Besides, we highlighted the importance of immune evasion in the process of BCR.

\section{DATA AVAILABILITY STATEMENT}

The original contributions presented in the study are included in the article/Supplementary Material, further inquiries can be directed to the corresponding author.

\section{AUTHOR CONTRIBUTIONS}

Conception: DF; data collection and processing: DF and XS; method and software: DF and XS; supervision: LY; manuscript draft: DF and XS; manuscript editing and review: all authors.

\section{FUNDING}

This program was supported by the National Natural Science Foundation of China (Grant Nos. 81974099, 82170785, 81974098, and 82170784), programs from the Science and Technology Department of Sichuan Province (Grant No. 21GJHZ0246), Young Investigator Award of Sichuan University 2017 (Grant No. 2017SCU04A17), Technology Innovation Research and Development Project of Chengdu Science and Technology Bureau (2019-YF05-00296-SN), and Sichuan University-Panzhihua Science and Technology Cooperation Special Fund (2020CDPZH-4).

\section{SUPPLEMENTARY MATERIAL}

The Supplementary Material for this article can be found online at: https://www.frontiersin.org/articles/10.3389/fcell.2022.803766/ full\#supplementary-material 


\section{REFERENCES}

Allen, G. W., Howard, A. R., Jarrard, D. F., and Ritter, M. A. (2007). Management of Prostate Cancer Recurrences after Radiation Therapy-Brachytherapy as a Salvage Option. Cancer 110 (7), 1405-1416. doi:10.1002/cncr.22940

Alvarez, S. W., Sviderskiy, V. O., Terzi, E. M., Papagiannakopoulos, T., Moreira, A. L., Adams, S., et al. (2017). NFS1 Undergoes Positive Selection in Lung Tumours and Protects Cells from Ferroptosis. Nature 551 (7682), 639-643. doi:10.1038/nature24637

Amling, C. L., Blute, M. L., Bergstralh, E. J., Seay, T. M., Slezak, J., and Zincke, H. (2000). Long-term hazard of Progression after Radical Prostatectomy for Clinically Localized Prostate Cancer: Continued Risk of Biochemical Failure after 5 Years. J. Urol. 164 (1), 101-105. doi:10.1097/00005392-20000700000023

Antonarakis, E. S., Feng, Z., Trock, B. J., Humphreys, E. B., Carducci, M. A., Partin, A. W., et al. (2012). The Natural History of Metastatic Progression in Men with Prostate-specific Antigen Recurrence after Radical Prostatectomy: Long-Term Follow-Up. BJU Int. 109 (1), 32-39. doi:10.1111/j.1464-410x.2011.10422.x

Aran, D., Sirota, M., and Butte, A. J. (2015). Systematic Pan-Cancer Analysis of Tumour Purity. Nat. Commun. 6, 8971. doi:10.1038/ncomms9971

Basuli, D., Tesfay, L., Deng, Z., Paul, B., Yamamoto, Y., Ning, G., et al. (2017). Iron Addiction: a Novel Therapeutic Target in Ovarian Cancer. Oncogene 36 (29), 4089-4099. doi:10.1038/onc.2017.11

Bebber, C. M., Müller, F., Prieto Clemente, L., Weber, J., and von Karstedt, S. (2020). Ferroptosis in Cancer Cell Biology. Cancers (Basel) 12 (1), e26476. doi:10.3390/cancers 12010164

Bersuker, K., Hendricks, J. M., Li, Z., Magtanong, L., Ford, B., Tang, P. H., et al. (2019). The CoQ Oxidoreductase FSP1 Acts Parallel to GPX4 to Inhibit Ferroptosis. Nature 575 (7784), 688-692. doi:10.1038/s41586-019-1705-2

Blake, S. J., Dougall, W. C., Miles, J. J., Teng, M. W. L., and Smyth, M. J. (2016). Molecular Pathways: Targeting CD96 and TIGIT for Cancer Immunotherapy. Clin. Cancer Res. 22 (21), 5183-5188. doi:10.1158/10780432.ccr-16-0933

Boorjian, S. A., Karnes, R. J., Crispen, P. L., Rangel, L. J., Bergstralh, E. J., and Blute, M. L. (2009). Radiation Therapy after Radical Prostatectomy: Impact on Metastasis and Survival. J. Urol. 182 (6), 2708-2715. doi:10.1016/j.juro.2009. 08.027

Charoentong, P., Finotello, F., Angelova, M., Mayer, C., Efremova, M., Rieder, D., et al. (2017). Pan-cancer Immunogenomic Analyses Reveal GenotypeImmunophenotype Relationships and Predictors of Response to Checkpoint Blockade. Cel Rep. 18 (1), 248-262. doi:10.1016/j.celrep.2016.12.019

Condeelis, J., and Pollard, J. W. (2006). Macrophages: Obligate Partners for Tumor Cell Migration, Invasion, and Metastasis. Cell 124 (2), 263-266. doi:10.1016/j. cell.2006.01.007

Danaher, P., Warren, S., Lu, R., Samayoa, J., Sullivan, A., Pekker, I., et al. (2018). Pan-Cancer Adaptive Immune Resistance as Defined by the Tumor Inflammation Signature (TIS): Results from the Cancer Genome Atlas (TCGA). J. Immunother. Cancer 6 (1), 63. doi:10.1186/s40425-018-0367-1

Das, U. N. (2019). Saturated Fatty Acids, MUFAs and PUFAs Regulate Ferroptosis. Cel. Chem. Biol. 26 (3), 309-311. doi:10.1016/j.chembiol.2019.03.001

Doll, S., Freitas, F. P., Shah, R., Aldrovandi, M., da Silva, M. C., Ingold, I., et al. (2019). FSP1 Is a Glutathione-independent Ferroptosis Suppressor. Nature 575 (7784), 693-698. doi:10.1038/s41586-019-1707-0

Dougall, W. C., Kurtulus, S., Smyth, M. J., and Anderson, A. C. (2017). TIGIT and CD96: New Checkpoint Receptor Targets for Cancer Immunotherapy. Immunol. Rev. 276 (1), 112-120. doi:10.1111/imr.12518

Du, M., Yuan, T., Schilter, K. F., Dittmar, R. L., Mackinnon, A., Huang, X., et al. (2015). Prostate Cancer Risk Locus at 8q24 as a Regulatory Hub by Physical Interactions with Multiple Genomic Loci across the Genome. Hum. Mol. Genet. 24 (1), 154-166. doi:10.1093/hmg/ddu426

Edgar, R., Domrachev, M., and Lash, A. E. (2002). Gene Expression Omnibus: NCBI Gene Expression and Hybridization Array Data Repository. Nucleic Acids Res. 30 (1), 207-210. doi:10.1093/nar/30.1.207

Epstein, J. I., Egevad, L., Amin, M. B., Delahunt, B., Srigley, J. R., and Humphrey, P. A. (2016). The 2014 International Society of Urological Pathology (ISUP) Consensus Conference on Gleason Grading of Prostatic Carcinoma. Am. J. Surg. Pathol. 40 (2), 244-252. doi:10.1097/pas.0000000000000530
Fiard, G., Stavrinides, V., Chambers, E. S., Heavey, S., Freeman, A., Ball, R., et al (2021). Cellular Senescence as a Possible Link between Prostate Diseases of the Ageing Male. Nat. Rev. Urol. 18 (10), 597-610. doi:10.1038/s41585-02100496-8

Ghoochani, A., Hsu, E.-C., Aslan, M., Rice, M. A., Nguyen, H. M., Brooks, J. D., et al. (2021). Ferroptosis Inducers Are a Novel Therapeutic Approach for Advanced Prostate Cancer. Cancer Res. 81 (6), 1583-1594. doi:10.1158/00085472.can-20-3477

Goldman, M. J., Craft, B., Hastie, M., Repečka, K., McDade, F., Kamath, A., et al. (2020). Visualizing and Interpreting Cancer Genomics Data via the Xena Platform. Nat. Biotechnol. 38 (6), 675-678. doi:10.1038/s41587-020-0546-8

Grivennikov, S. I., Greten, F. R., and Karin, M. (2010). Immunity, Inflammation, and Cancer. Cell 140 (6), 883-899. doi:10.1016/j.cell.2010.01.025

Han, H., Cho, J.-W., Lee, S., Yun, A., Kim, H., Bae, D., et al. (2018). TRRUST V2: an Expanded Reference Database of Human and Mouse Transcriptional Regulatory Interactions. Nucleic Acids Res. 46 (D1), D380-D386. doi:10. 1093/nar/gkx1013

Hanahan, D., and Weinberg, R. A. (2011). Hallmarks of Cancer: the Next Generation. Cell 144 (5), 646-674. doi:10.1016/j.cell.2011.02.013

Hart, I. R., and Fidler, I. J. (1980). Role of Organ Selectivity in the Determination of Metastatic Patterns of B16 Melanoma. Cancer Res. 40 (7), 2281-2287.

Hayano, M., Yang, W. S., Corn, C. K., Pagano, N. C., and Stockwell, B. R. (2016). Loss of Cysteinyl-tRNA Synthetase (CARS) Induces the Transsulfuration Pathway and Inhibits Ferroptosis Induced by Cystine Deprivation. Cel. Death Differ. 23 (2), 270-278. doi:10.1038/cdd.2015.93

Hu, X., Feng, H., Huang, H., Gu, W., Fang, Q., Xie, Y., et al. (2019). Downregulated Long Noncoding RNA PART1 Inhibits Proliferation and Promotes Apoptosis in Bladder Cancer. Technol. Cancer Res. Treat. 18, 1533033819846638. doi:10. $1177 / 1533033819846638$

Hull, G. W., Rabbani, F., Abbas, F., Wheeler, T. M., Kattan, M. W., and Scardino, P. T. (2002). Cancer Control with Radical Prostatectomy Alone in 1,000 Consecutive Patients. J. Urol. 167 (2 Pt 1), 528-534. doi:10.1097/00005392200202000-00018

Jain, S., Lyons, C. A., Walker, S. M., McQuaid, S., Hynes, S. O., Mitchell, D. M., et al. (2018). Validation of a Metastatic Assay Using Biopsies to Improve Risk Stratification in Patients with Prostate Cancer Treated with Radical Radiation Therapy. Ann. Oncol. 29 (1), 215-222. doi:10.1093/annonc/mdx637

Jelinek, A., Heyder, L., Daude, M., Plessner, M., Krippner, S., Grosse, R., et al. (2018). Mitochondrial rescue Prevents Glutathione Peroxidase-dependent Ferroptosis. Free Radic. Biol. Med. 117, 45-57. doi:10.1016/j.freeradbiomed. 2018.01.019

Jeong, H. M., Kim, R. N., Kwon, M. J., Oh, E., Han, J., Lee, S. K., et al. (2017) Targeted Exome Sequencing of Korean Triple-Negative Breast Cancer Reveals Homozygous Deletions Associated with Poor Prognosis of Adjuvant Chemotherapy-Treated Patients. Oncotarget 8 (37), 61538-61550. doi:10. 18632/oncotarget.18618

Joyce, J. A., and Pollard, J. W. (2009). Microenvironmental Regulation of Metastasis. Nat. Rev. Cancer 9 (4), 239-252. doi:10.1038/nrc2618

Junttila, M. R., and de Sauvage, F. J. (2013). Influence of Tumour Microenvironment Heterogeneity on Therapeutic Response. Nature 501 (7467), 346-354. doi:10.1038/nature12626

Koukourakis, M. I., Giatromanolaki, A., Panteliadou, M., Pouliliou, S. E., Chondrou, P. S., Mavropoulou, S., et al. (2014). Lactate Dehydrogenase 5 Isoenzyme Overexpression Defines Resistance of Prostate Cancer to Radiotherapy. Br. J. Cancer 110 (9), 2217-2223. doi:10.1038/bjc.2014.158

Kuner, R., Fälth, M., Pressinotti, N. C., Brase, J. C., Puig, S. B., Metzger, J., et al. (2013). The Maternal Embryonic Leucine Zipper Kinase (MELK) Is Upregulated in High-Grade Prostate Cancer. J. Mol. Med. 91 (2), 237-248. doi:10.1007/s00109-012-0949-1

Lachaier, E., Louandre, C., Godin, C., Saidak, Z., Baert, M., Diouf, M., et al. (2014). Sorafenib Induces Ferroptosis in Human Cancer Cell Lines Originating from Different Solid Tumors. Anticancer Res. 34 (11), 6417-6422.

Li, X. R., Zhou, K. Q., Yin, Z., Gao, Y. L., and Yang, X. (2020). Knockdown of FBP1 Enhances Radiosensitivity in Prostate Cancer Cells by Activating Autophagy. Neoplasma 67 (5), 982-991. doi:10.4149/neo_2020_190807N728

Liberzon, A., Subramanian, A., Pinchback, R., Thorvaldsdottir, H., Tamayo, P., and Mesirov, J. P. (2011). Molecular Signatures Database (MSigDB) 3.0. Bioinformatics 27 (12), 1739-1740. doi:10.1093/bioinformatics/btr260 
Lin, R., Zhang, Z., Chen, L., Zhou, Y., Zou, P., Feng, C., et al. (2016). Dihydroartemisinin (DHA) Induces Ferroptosis and Causes Cell Cycle Arrest in Head and Neck Carcinoma Cells. Cancer Lett. 381 (1), 165-175. doi:10.1016/j.canlet.2016.07.033

Liu, C.-J., Hu, F.-F., Xia, M.-X., Han, L., Zhang, Q., and Guo, A.-Y. (2018). GSCALite: a Web Server for Gene Set Cancer Analysis. Bioinformatics 34 (21), 3771-3772. doi:10.1093/bioinformatics/bty411

Liu, B., Ma, T., Li, Q., Wang, S., Sun, W., Li, W., et al. (2019). Identification of a lncRNAassociated Competing Endogenous RNAregulated Network in clear Cell Renal Cell Carcinoma. Mol. Med. Rep. 20 (1), 485-494. doi:10.3892/mmr. 2019.10290

Liu, F., Huang, J., He, F., Ma, X., Fan, F., Meng, M., et al. (2020). CD96, a New Immune Checkpoint, Correlates with Immune Profile and Clinical Outcome of Glioma. Sci. Rep. 10 (1), 10768. doi:10.1038/s41598-020-66806-Z

Lv, Z., Wang, J., Wang, X., Mo, M., Tang, G., Xu, H., et al. (2021). Identifying a Ferroptosis-Related Gene Signature for Predicting Biochemical Recurrence of Prostate Cancer. Front. Cel Dev. Biol. 9, 666025. doi:10.3389/fcell.2021.666025

Maas, R. J., Hoogstad-van Evert, J. S., Van der Meer, J. M., Mekers, V., Rezaeifard, S., Korman, A. J., et al. (2020). TIGIT Blockade Enhances Functionality of Peritoneal NK Cells with Altered Expression of DNAM-1/TIGIT/CD96 Checkpoint Molecules in Ovarian Cancer. Oncoimmunology 9 (1), 1843247. doi:10.1080/2162402x.2020.1843247

Magtanong, L., Ko, P.-J., To, M., Cao, J. Y., Forcina, G. C., Tarangelo, A., et al. (2019). Exogenous Monounsaturated Fatty Acids Promote a FerroptosisResistant Cell State. Cel Chem. Biol. 26 (3), 420-432. doi:10.1016/j.chembiol. 2018.11.016

Mantovani, A., Allavena, P., Sica, A., and Balkwill, F. (2008). Cancer-related Inflammation. Nature 454 (7203), 436-444. doi:10.1038/nature07205

Mittal, D., Lepletier, A., Madore, J., Aguilera, A. R., Stannard, K., Blake, S. J., et al. (2019). CD96 Is an Immune Checkpoint that Regulates CD8+ T-Cell Antitumor Function. Cancer Immunol. Res. 7 (4), 559-571. doi:10.1158/ 2326-6066.cir-18-0637

Montanari, M., Rossetti, S., Cavaliere, C., D’Aniello, C., Malzone, M. G., Vanacore, D., et al. (2017). Epithelial-mesenchymal Transition in Prostate Cancer: an Overview. Oncotarget 8 (21), 35376-35389. doi:10.18632/oncotarget.15686

Obinata, D., Takayama, K., Fujiwara, K., Suzuki, T., Tsutsumi, S., Fukuda, N., et al. (2016). Targeting Octl Genomic Function Inhibits Androgen Receptor Signaling and Castration-Resistant Prostate Cancer Growth. Oncogene 35 (49), 6350-6358. doi:10.1038/onc.2016.171

Odero-Marah, V., Hawsawi, O., Henderson, V., and Sweeney, J. (2018). EpithelialMesenchymal Transition (EMT) and Prostate Cancer. Adv. Exp. Med. Biol. 1095, 101-110. doi:10.1007/978-3-319-95693-0_6

Paget, S. (1989). The Distribution of Secondary Growths in Cancer of the Breast. 1889. Cancer Metastasis Rev. 8 (2), 98-101.

Påhlman, S., and Mohlin, S. (2018). Hypoxia and Hypoxia-Inducible Factors in Neuroblastoma. Cel. Tissue Res. 372 (2), 269-275. doi:10.1007/s00441-0172701-1

Paraskevopoulou, M. D., Vlachos, I. S., Karagkouni, D., Georgakilas, G., Kanellos, I., Vergoulis, T., et al. (2016). DIANA-LncBase V2: Indexing microRNA Targets on Non-coding Transcripts. Nucleic Acids Res. 44 (D1), D231-D238. doi:10.1093/nar/gkv1270

Peng, Y., Cui, C., He, Y., Ouzhuluobu, Zhang, H., Yang, D., et al. (2017). DownRegulation of EPAS1 Transcription and Genetic Adaptation of Tibetans to High-Altitude Hypoxia. Mol. Biol. Evol. 34 (4), 818-830. doi:10.1093/molbev/ msw280

Penney, K. L., Sinnott, J. A., Tyekucheva, S., Gerke, T., Shui, I. M., Kraft, P., et al. (2015). Association of Prostate Cancer Risk Variants with Gene Expression in normal and Tumor Tissue. Cancer Epidemiol. Biomark. Prev. 24 (1), 255-260. doi:10.1158/1055-9965.epi-14-0694-t

Pound, C. R., Partin, A. W., Eisenberger, M. A., Chan, D. W., Pearson, J. D., and Walsh, P. C. (1999). Natural History of Progression after PSA Elevation Following Radical Prostatectomy. JAMA 281 (17), 1591-1597. doi:10.1001/ jama.281.17.1591

Racle, J., de Jonge, K., Baumgaertner, P., Speiser, D. E., and Gfeller, D. (2017). Simultaneous Enumeration of Cancer and Immune Cell Types from Bulk Tumor Gene Expression Data. Elife 6, e26476. doi:10.7554/eLife.26476

Roehl, K. A., Han, M., Ramos, C. G., Antenor, J. A. V., and Catalona, W. J. (2004). Cancer Progression and Survival Rates Following Anatomical Radical
Retropubic Prostatectomy in 3,478 Consecutive Patients: Long-Term Results. J. Urol. 172 (3), 910-914. doi:10.1097/01.ju.0000134888.22332.bb

Rohr-Udilova, N., Bauer, E., Timelthaler, G., Eferl, R., Stolze, K., Pinter, M., et al. (2018). Impact of Glutathione Peroxidase 4 on Cell Proliferation, Angiogenesis and Cytokine Production in Hepatocellular Carcinoma. Oncotarget 9 (11), 10054-10068. doi:10.18632/oncotarget.24300

Shimada, K., Skouta, R., Kaplan, A., Yang, W. S., Hayano, M., Dixon, S. J., et al. (2016). Global Survey of Cell Death Mechanisms Reveals Metabolic Regulation of Ferroptosis. Nat. Chem. Biol. 12 (7), 497-503. doi:10.1038/nchembio.2079

Sica, A., Allavena, P., and Mantovani, A. (2008). Cancer Related Inflammation: the Macrophage Connection. Cancer Lett. 267 (2), 204-215. doi:10.1016/j.canlet. 2008.03.028

Siegel, R. L., Miller, K. D., Fuchs, H. E., and Jemal, A. (2021). Cancer Statistics, 2021. CA A. Cancer J. Clin. 71 (1), 7-33. doi:10.3322/caac.21654

Sinnott, J. A., Peisch, S. F., Tyekucheva, S., Gerke, T., Lis, R., Rider, J. R., et al. (2017). Prognostic Utility of a New mRNA Expression Signature of Gleason Score. Clin. Cancer Res. 23 (1), 81-87. doi:10.1158/1078-0432.ccr-16-1245

Soucek, L., Lawlor, E. R., Soto, D., Shchors, K., Swigart, L. B., and Evan, G. I. (2007). Mast Cells Are Required for Angiogenesis and Macroscopic Expansion of MycInduced Pancreatic Islet Tumors. Nat. Med. 13 (10), 1211-1218. doi:10.1038/ nm1649

Stelzer, G., Rosen, N., Plaschkes, I., Zimmerman, S., Twik, M., Fishilevich, S., et al. (2016). The GeneCards Suite: From Gene Data Mining to Disease Genome Sequence Analyses. Curr. Protoc. Bioinf. 54, 1-33. doi:10.1002/cpbi.5

Sticht, C., De La Torre, C., Parveen, A., and Gretz, N. (2018). miRWalk: An Online Resource for Prediction of microRNA Binding Sites. PLoS One 13 (10), e0206239. doi:10.1371/journal.pone.0206239

Stockwell, B. R., Friedmann Angeli, J. P., Bayir, H., Bush, A. I., Conrad, M., Dixon, S. J., et al. (2017). Ferroptosis: A Regulated Cell Death Nexus Linking Metabolism, Redox Biology, and Disease. Cell 171 (2), 273-285. doi:10.1016/ j.cell.2017.09.021

Sun, X., Ou, Z., Xie, M., Kang, R., Fan, Y., Niu, X., et al. (2015). HSPB1 as a Novel Regulator of Ferroptotic Cancer Cell Death. Oncogene 34 (45), 5617-5625. doi:10.1038/onc.2015.32

Sun, S.-Q., Gu, X., Gao, X.-S., Li, Y., Yu, H., Xiong, W., et al. (2016). Overexpression of AKR1C3 Significantly Enhances Human Prostate Cancer Cells Resistance to Radiation. Oncotarget 7 (30), 48050-48058. doi:10.18632/oncotarget.10347

Sun, H., Huang, Q., Huang, M., Wen, H., Lin, R., Zheng, M., et al. (2019). Human CD96 Correlates to Natural Killer Cell Exhaustion and Predicts the Prognosis of Human Hepatocellular Carcinoma. Hepatology 70 (1), 168-183. doi:10.1002/ hep. 30347

Tripathi, M., Billet, S., and Bhowmick, N. A. (2012). Understanding the Role of Stromal Fibroblasts in Cancer Progression. Cell Adhes. Migration 6 (3), 231-235. doi:10.4161/cam.20419

Ubellacker, J. M., Tasdogan, A., Ramesh, V., Shen, B., Mitchell, E. C., MartinSandoval, M. S., et al. (2020). Lymph Protects Metastasizing Melanoma Cells from Ferroptosis. Nature 585 (7823), 113-118. doi:10.1038/s41586-020-2623-Z

Van den Broeck, T., van den Bergh, R. C. N., Arfi, N., Gross, T., Moris, L., Briers, E., et al. (2019). Prognostic Value of Biochemical Recurrence Following Treatment with Curative Intent for Prostate Cancer: A Systematic Review. Eur. Urol. 75 (6), 967-987. doi:10.1016/j.eururo.2018.10.011

Wallis, C. J. D., Saskin, R., Choo, R., Herschorn, S., Kodama, R. T., Satkunasivam, R., et al. (2016). Surgery versus Radiotherapy for Clinically-Localized Prostate Cancer: A Systematic Review and Meta-Analysis. Eur. Urol. 70 (1), 21-30. doi:10.1016/j.eururo.2015.11.010

Wallis, C. J. D., Glaser, A., Hu, J. C., Huland, H., Lawrentschuk, N., Moon, D., et al. (2018). Survival and Complications Following Surgery and Radiation for Localized Prostate Cancer: An International Collaborative Review. Eur. Urol. 73 (1), 11-20. doi:10.1016/j.eururo.2017.05.055

Warde-Farley, D., Donaldson, S. L., Comes, O., Zuberi, K., Badrawi, R., Chao, P., et al. (2010). The GeneMANIA Prediction Server: Biological Network Integration for Gene Prioritization and Predicting Gene Function. Nucleic Acids Res. 38 (Web Server issue), W214-W220. doi:10.1093/nar/gkq537

Weber, D. C., Wang, H., Cozzi, L., Dipasquale, G., Khan, H. G., Ratib, O., et al. (2009). RapidArc, Intensity Modulated Photon and Proton Techniques for Recurrent Prostate Cancer in Previously Irradiated Patients: a Treatment Planning Comparison Study. Radiat. Oncol. 4, 34. doi:10.1186/1748-717x4-34 
Wiegel, T., Lohm, G., Bottke, D., Höcht, S., Miller, K., Siegmann, A., et al. (2009). Achieving an Undetectable PSA after Radiotherapy for Biochemical Progression after Radical Prostatectomy Is an Independent Predictor of Biochemical Outcome-Results of a Retrospective Study. Int. J. Radiat. Oncol. ${ }^{\star}$ Biol. ${ }^{\star}$ Physics 73 (4), 1009-1016. doi:10.1016/j.ijrobp.2008.06.1922

Wu, Z., Shi, J., Lai, C., Li, K., Li, K., Li, Z., et al. (2021). Clinicopathological Significance and Prognostic Value of Cancer-Associated Fibroblasts in Prostate Cancer Patients. Urol. Oncol. 39 (7), 433.e17-433.e23. doi:10.1016/j.urolonc. 2021.05.004

Xie, X., Xu, Z., Wang, C., Fang, C., Zhao, J., Xu, L., et al. (2018). Tip60 Is Associated with Resistance to X-ray Irradiation in Prostate Cancer. FEBS Open Bio 8 (2), 271-278. doi:10.1002/2211-5463.12371

Xu, T., Ding, W., Ji, X., Ao, X., Liu, Y., Yu, W., et al. (2019). Molecular Mechanisms of Ferroptosis and its Role in Cancer Therapy. J. Cel. Mol. Med. 23 (8), 4900-4912. doi:10.1111/jcmm.14511

Yan, Z., Xiao, Y., Chen, Y., and Luo, G. (2020). Screening and Identification of Epithelial-To-Mesenchymal Transition-Related circRNA and miRNA in Prostate Cancer. Pathol. - Res. Pract. 216 (2), 152784. doi:10.1016/j.prp. 2019.152784

Yang, W. S., SriRamaratnam, R., Welsch, M. E., Shimada, K., Skouta, R., Viswanathan, V. S., et al. (2014). Regulation of Ferroptotic Cancer Cell Death by GPX4. Cell 156 (1-2), 317-331. doi:10.1016/j.cell.2013.12.010

Ye, W., Luo, C., Liu, F., Liu, Z., and Chen, F. (2021). CD96 Correlates with Immune Infiltration and Impacts Patient Prognosis: A Pan-Cancer Analysis. Front. Oncol. 11, 634617. doi:10.3389/fonc.2021.634617

Yi, J., Zhu, J., Wu, J., Thompson, C. B., and Jiang, X. (2020). Oncogenic Activation of PI3K-AKT-mTOR Signaling Suppresses Ferroptosis via SREBP-Mediated Lipogenesis. Proc. Natl. Acad. Sci. USA 117 (49), 31189-31197. doi:10.1073/ pnas.2017152117

Yoshihara, K., Shahmoradgoli, M., Martínez, E., Vegesna, R., Kim, H., TorresGarcia, W., et al. (2013). Inferring Tumour Purity and Stromal and Immune Cell Admixture from Expression Data. Nat. Commun. 4, 2612. doi:10.1038/ ncomms 3612

Zeng, D., Ye, Z., Shen, R., Yu, G., Wu, J., Xiong, Y., et al. (2021). IOBR: MultiOmics Immuno-Oncology Biological Research to Decode Tumor
Microenvironment and Signatures. Front. Immunol. 12, 687975. doi:10. 3389/fimmu.2021.687975

Zhang, Q., Zhong, H., Fan, Y., Liu, Q., Song, J., Yao, S., et al. (2020). Immune and Clinical Features of CD96 Expression in Glioma by In Silico Analysis. Front. Bioeng. Biotechnol. 8, 592. doi:10.3389/fbioe.2020.00592

Zhao, S. G., Lehrer, J., Chang, S. L., Das, R., Erho, N., Liu, Y., et al. (2019). The Immune Landscape of Prostate Cancer and Nomination of PD-L2 as a Potential Therapeutic Target. J. Natl. Cancer Inst. 111 (3), 301-310. doi:10.1093/jnci/ djy141

Zhou, M., Zhang, Z., Bao, S., Hou, P., Yan, C., Su, J., et al. (2021). Computational Recognition of lncRNA Signature of Tumor-Infiltrating B Lymphocytes with Potential Implications in Prognosis and Immunotherapy of Bladder Cancer. Brief Bioinform. 22 (3), bbaa047. doi:10.1093/bib/bbaa047

Zou, Y., Palte, M. J., Deik, A. A., Li, H., Eaton, J. K., Wang, W., et al. (2019). A GPX4-dependent Cancer Cell State Underlies the clear-cell Morphology and Confers Sensitivity to Ferroptosis. Nat. Commun. 10 (1), 1617. doi:10.1038/ s41467-019-09277-9

Conflict of Interest: The authors declare that the research was conducted in the absence of any commercial or financial relationships that could be construed as a potential conflict of interest.

Publisher's Note: All claims expressed in this article are solely those of the authors and do not necessarily represent those of their affiliated organizations, or those of the publisher, the editors, and the reviewers. Any product that may be evaluated in this article, or claim that may be made by its manufacturer, is not guaranteed or endorsed by the publisher.

Copyright (C) 2022 Feng, Shi, Xiong, Zhang, Li, Wei and Yang. This is an open-access article distributed under the terms of the Creative Commons Attribution License (CC $B Y)$. The use, distribution or reproduction in other forums is permitted, provided the original author(s) and the copyright owner(s) are credited and that the original publication in this journal is cited, in accordance with accepted academic practice. No use, distribution or reproduction is permitted which does not comply with these terms. 